\title{
Contents, Vol. 18, 1907
}

\section{Inhalts -Verzeichnis.}

Original-Arbeiten.

Amberg, Hans, Weiterer kasuistischer Beitrag zur Ent-fernung von Eisensplittern aus dem Auge rait demInnenpolmagneten 511

Ask, Fritz, Beraerkungen zur Myopiefrage 47

Bach, L., Allgemeine Pathologie der Pupille 499

Bartels, Martin, Einige Bemerkungen uncl Erfahrungen überdie Rolle der sogenannten

Neurosen, speziell der Psycho-neurosen, Hysteric und Neurasthenie in der Augen-heilkunde 399, 522

Gherno, Franz, Ueber die unter dem Nam en Blepharitis

ciliaris bekannten Erkrankungen des Lidrandes ... 1

Cohen, Curt, Erwiderung auf die Arbeit von Krauss: Ueber

Veränderungen am Sehorgan bei Schädelverbildungen 545

Enslín, Fr., Erwiderung auf die Arbeit von Krauss: Ueber

Veränderungen am Sehorgan bei Schädelverbildungen 542

Hamburger, C, Zu dem Aufsatze Asks; Bemerkungen zur

Myopiefrage 539

Hesse, Robert, 500 Kataraktextraktionen aus der Grazer

Augenklinik 21

Köllner, H., Ueber den Einfluss der Refraktionsanomalienauf die Farbenwahrnehmung, besonders auf die Be-urteilung spektraler Gleichungen $\quad 430$

Koster-Gzn. W'., Entfernung einer bei der Extraktion in den

Glaskörper luxierten Linse nach 7 Jahren 220

- - Eine Fixationspincette 521

Krauss, W., Bemerkungen zu den Erwiderungen von Enslin

und Cohen auf meine dort angegebene Arbeit .... 547

Krusius, Franz F., Zur diagnostischen Verwertung des Eserins

bei Pupillenstörungen 442

Kuhnt, Hermann, Ueber Uebertragung von Hautschollen

nach der Enukleation bei eingeengtem Bindehautsack 152

Kuschel, J., Die Arehitektur des Auges, ein Regulierungs-mechanismus für die intraokularen Druck- und Strom-schwankungen 116, 316

Laas, Otto, Zwei Eälle von kontralateraler Sehstörung nachOperation der Spina septi narium, der eine kompliziertdurch Erblindung auf der Seite der Operation und(lurch meningitische Symptome 142

L·auber, H., Ein Fall von Narbenfixation der Augen nachluetischer Nekrose der nasalen

Orbitalwand. (HierzuTafel I) 41

Michel, J. v., Ueber die pathologiseh-anatomischen Veränderungen der Blutgefässe des

Augapfels bei Syphilis.(Hierzu Tafel III) 
Napp, Otto, Untersuchungen über den Bakteriengehalt derßindehautsäcke von Staroperierten bei Anwendungaseptiseher Verbände 198

zur Nedden, Max, Untersuchungen über das Vorkommen bak-terizider Substanzen im Bindehautsekret nebst Bemerkungen über den Heilungsprozess der Bindehaut-katarrhe $\quad 300$ Peters, A., Die Pathologie und Therapie der einfachen chronischen Konjunktivitisformen415

$-\mathrm{IV}-$

Pinctis, Fríedrich, Spontanheilung eines traumatischen pul-

sierenden Exophthalmus 33

Schmidt, Heinrich, Zur Lösung des Problems der Kugel-

einheilung 137

Steiger, Adolf, Die Veränderungen des Hornhautastigmatis-mus und der Hornhautrefraktion im

Zeitraum von 5V3 Jahren an Hand von 3000 Doppelmessungen 103, 223

Twietmeyer, J., Zur Kasuistik der zentralen Lochbildung . 44?

Ulbrich, H., Beobachtungen über Altersstar mit Sekundär-

glaukom 133

Verderame, Ph., Experimentelle Untersuchungen über die gewebsschädigenden Eigenschaften des Novocains . . 191

Vüllers, H., Neue, diagnostisch wichtige Resultate bei Durch-leuchtung des Auges mit der

Sachsschen Durchleuch-tungslampe. (Hierzu Tafel II) 215

Bericht über die deutsche ophthalmologische Literatur.

Diagnostik und Extraktion von Fremdkörpern. Von Dr.

E. Asmus in Düsseldorf 70

Untersuchungsmethoden (I. u. II. Sem. 1906). Von Prof.

Dr. Dimmer in Graz 335

Physiologie des Gesichtssinns (1906). Von Priv.-Doz. Dr.

Nicolai in Berlin 55, 154

Anatomie des Auges. (II. Semester 1906.) Von Professor

Dr. Joh. Sobotta in Würzburg 548

Bericht über die ausländische ophthalmologische Literatur.

Bericht über die englische ophthalmologische Literatur. Von

Dr. Gruber in London 347

Bericht über die holländische ophthalmologische Literatur.(II. Semester 1906.) Von Dr. Q. J.

Schoute in Amsterdamund Prof. W. Koster-Gzn. in Leiden 569

Bericht über die russische ophthalmologische Literatur. Von

Dr. Th. Wemcke in Odessa 236, 450

Bericht über die ungarisclie ophthalmologische Literatur(I. u. II. Sem. 1906). Von Prof. Dr. von

Grósz undPriv.-Doz. Dr. Leitner in Budapest472

Gesellschaftsberichte.

79. Naturforscherversammlung in Dresden 1907354

Bericht über die XXXIV. Zusammenkunft der ophthal-

mologisclien Gesellschaft in Heidelberg 255

Bericht über die III. Versammlung der ungarischen ophthal-

mologischen Gesellschaft in Budapest

79

Société d'Ophthalmologie de Paris 480

Ophthalmologische Gesellschaft in Wien 284 
Unfall- und Versicherungskunde $\quad 94,491,574$

Therapeutische Umschau 95, 180, 290, 387, 492, 575

Offene Korrespondenz 293, 539

Tagesgeschichtliches und Notizen 98, 294, 498, 582

Buchanzeigen 184

Literaturverzeichnis 100, 187, 392, 495, 579

S. Sachregister583

Namenregister 592 